\title{
Deep Nuclear Resonant Tunneling Thermal Rate Constant Calculations
}

\author{
Salvatore Mandrà \\ Dipartimento di Fisica, Università degli Studi di Milano, \\ via Celoria 16, 20133 Milano, Italy* \\ Stéphanie Valleau \\ Department of Chemistry and Chemical Biology, \\ Harvard University, 12 Oxford Street, \\ Cambridge, Massachusetts 02138, USA \\ Michele Ceotto \\ Dipartimento di Chimica, Università degli Studi \\ di Milano, via Golgi 19, 20133 Milano, Italy
}

(Dated: 18/09/2012)

\begin{abstract}
A fast and robust time-independent method for calculating thermal rate constants in the deep resonant tunneling regime for scattering reactions is presented. The method is based on the calculation of cumulative reaction probabilities which are integrated to give thermal rate constants. We tested our method with both continuous (Eckart barrier) and discontinuous (double rectangular barrier) first derivative potentials. Our results show that the presented method is robust enough to deal with extreme resonating conditions such as multiple barrier potentials. Finally, the calculation of the thermal rate constant for double Eckart potentials with several metastable states is reported.
\end{abstract}

*Electronic address: salvatore.mandra@unimi.it

${ }^{\dagger}$ Electronic address: michele.ceotto@unimi.it 


\section{INTRODUCTION}

The thermal rate constant $k(T)$ is an essential observable for the quantitative description of chemical dynamics and its study offers a common playground of comparison between innovative experimental techniques, new theories and computational tools. Since the formulation of the transition state theory [1-3], many efforts have been devoted to the development of an exact and computationally feasible approach to the thermal rate constant calculation. In this paper, we focus on the calculation of $k(T)$ for double barrier passages in the presence of several quasi-bound (resonant) states. The importance of studying $k(T)$ in the presence of quasi-bound states is related to the occurrence of resonant tunneling, which considerably increases the transmission probability at the resonance energy. Specifically, resonant tunneling occurs when an incident wave-packet has an energy which is comparable to one of the quasi-bound state energies: in this case, the tunneling probability is greatly enhanced and the wave-packet can cross the potential without an effective reduction of its amplitude. Pioneering experimental observations of resonant tunneling lead to what is presently known as Ramsauer's effect (or negative resistance). In particular, such effect manifested itself in the form of electrons crossing double-barrier structures having a thin GaAs film sandwiched between two GaAlAs barriers [4,5]. The resonance was observed from peaks in the tunneling current, when the voltage was set near the quasi-stationary energy states of the potential well. Beyond semiconductors [6], resonant tunneling is relevant to describe several experimental systems such as quantum dots [7], the Fabry-Perot interferometer [8] and molecular reactions [9-12]. Interestingly, resonances are weaker when tunneling is coupled to an applied field [13]. Resonances have also been calculated for collinear reactions in the presence of a single barrier and van der Waals wells $[14,15]$. Another example of molecular resonant scattering is the reaction $\mathrm{H}+\mathrm{O}_{2}[11,12]$, where the resonances are induced by the presence of an intermediate well. In this case, full dimensional accurate quantum results are available $[16,17]$.

Although many analytical results can be obtained for single barrier potentials (e.g. the rectangular barrier or Eckart barrier [18]), little is known about resonant tunneling for potentials with two or more barriers beyond the simple case of double rectangular potentials [19-22]. This is due to the intensive numerical effort required to compute $k(T)$ exactly, even in the one dimensional case. Moreover, these calculations are much more challenging 
than evaluating the transmission probability for systems where resonance is induced by the presence of an intermediate well [17] (with fixed total angular momentum) or for single barrier potentials in the presence of wells. [15]

Calculations of $k(T)$ can be done using either a time-dependent or time-independent method. In the time-dependent approach, a non-negligible amount of computational time is required due to the presence of long-lived resonant states . Further, a large spatial width wave-packet is needed in order to observe resonances which is costly to in terms of grid methods. The corresponding momentum is well-defined and it has a small energy spread. From this point of view, double barrier potentials can also be used as an energy filter to get those wave-packet components whose momenta distribution width is comparable with the resonance width. Inspired by this consideration, Moyesev and co-workers have developed a non-Hermitian representation of the resonance states decay and solved the time-dependent Schrödinger equation with outgoing boundary conditions [23]. They were able to calculate the tunneling transmission probability for two Gaussian barriers supporting up to five resonance states.

Major advances in the time-dependent picture have been obtained by Miller and coworkers $[24,25]$, who developed a method in which a time integration of the flux auto-correlation function is used to compute $k(T)$ directly. Although this method has been widely used [25] , it is practically limited to potentials with one single barrier or two narrow barriers [27]. Indeed, when many quasi-bound states are present, the numerical effort required to compute the long-time dynamics for the thermal rate constant increases greatly. In this direction, Peskin et al. [28-30] developed a more stable variant of the flux auto-correlation method to compute $k(T)$ even in the presence of resonances, the so called Flux Averaging method (FAM). Although this last method partially solves the issue of the long-time dynamics imposed by the flux auto-correlation approach, the computational time required to reach convergence still dramatically increases in the deep resonant regime.

To overcome these limitations, in this paper we present a time-independent approach to compute $k(T)$ for any arbitrary multi-barrier potential (even for first-derivative discontinuous potentials), in the presence of many quasi-bound states. The method consists in a fast and robust procedure for calculating $k(T)$ as the thermal average of the transmission probability $T(E)$, namely the probability of a quantum particle to cross the scattering potential, 
by directly solving the Schrödinger equation as an ordinary differential equation (ODE) with the energy as a parameter. This avoids having to solve for an eigenvalue problem. Although the thermal rate constant has been computed for potentials with few resonant peaks [27, 29], to the best of our knowledge $k(T)$ has not been evaluated in the presence of two clearly separated barriers with several quasi-bound states. Although our method is designed for a one dimensional potential, in principle it could be extended to an arbitrary number of dimensions with an additional numerical effort.

The paper is organized as follows. In Section II the time-dependent flux and the FAM methods for the thermal rate constant calculation are briefly recalled. In Section III we present our time-independent approach and in Section IV we compare it to the timedependent approach by applying it to a series of potential energy barriers including barriers with many quasi bound states. We summarize by discussing our findings in Section V and conclude in the last Section.

\section{THE TIME-DEPENDENT APPROACH}

The thermal rate constant $k(T)$ for an arbitrary system at a given temperature $T$ can be written as

$$
k(T)=\frac{1}{Q_{R}(T)} \int_{0}^{\infty} C_{i, j}(t) d t
$$

where $Q_{R}(T)$ is the reactants partition function and, as first derived by Miller, Schwartz and Tromp [24], the flux auto-correlation function $C_{i, j}(t)$ can be expressed as

$$
C_{i, j}(t)=\operatorname{Tr}\left[e^{-\beta \hat{H} / 2} \hat{F}_{i} e^{-\beta \hat{H} / 2} e^{i \hat{H} t / \hbar} \hat{F}_{j} e^{-i \hat{H} t / \hbar}\right],
$$

where $\beta=\frac{1}{k_{b} T}$ and $\hat{H}$ is the system's Hamiltonian. The flux operator $\hat{F}_{i}$

$$
\hat{F}_{i}=\frac{i}{\hbar}\left[\hat{H}, \hat{h}\left(f_{i}(\mathbf{s})\right)\right]=\frac{d}{d t} \hat{h}\left(f_{i}(\mathbf{s})\right)
$$

is defined respect to a dividing surface $f_{i}(\mathbf{s})=0$, placed between reactants and products and $\mathbf{s}$ is the vector of coordinates. $\hat{h}$ is a Heaviside operator and it has expectation value $1(0)$ on the product (reactant) side. For a dividing surface loci equation of the type $f_{i}(\mathbf{s})=\mathbf{s}-\mathbf{s}_{i}=0$, $\hat{F}_{i}$ assumes the explicit expression

$$
\hat{F}_{i}=\frac{1}{2 m}\left[\hat{\mathbf{p}} \delta\left(\mathbf{s}-\mathbf{s}_{i}\right)+\delta\left(\mathbf{s}-\mathbf{s}_{i}\right) \hat{\mathbf{p}}\right],
$$


where $m$ is the mass of the particle and $\hat{\mathbf{p}}$ its momentum operator. Therefore, the reaction rate is obtained by evaluating both flux operators with respect to their dividing surfaces and then by propagating one of them up to a long enough time to obtain a complete decay of the auto-correlation function. One can appreciate how Eq. (1) is an exact expression for the thermal rate constant in any dimension and for any system, including complex condensed phase systems [31-49]. However, when the reaction profile involves potential wells or multiple barrier passage, the application of Eq. (1) becomes quite cumbersome. Typical examples are reactions of the type $\mathrm{OH}+\mathrm{O} \rightarrow \mathrm{H}+\mathrm{O}_{2}$ [10] and the ketene isomerization [9]. In these cases, dividing surfaces are conveniently placed one on the reactant side and another on the product side. However, the long-time dynamics associated with the presence of metastable states leads to slow convergence in the time integration of Eq. (1). Finally, the flux autocorrelation function fails to converge in a reasonable amount of time due to the presence of long-lived resonance states.

A variant of the Miller, Swartz and Tromp's formula, Eq. (1), for resonating systems has been put forward by Peskin et al. [28]. Such variant is called the "flux averaging" method (FAM) and it allows the calculation of the thermal rate constants for double barrier potentials. The method takes advantage of the fact that, after a certain amount of time, the ratio of the fluxes in entrance to fluxes in exit channels is roughly constant. Once the ratio is defined, one can obtain the asymptotic-time limit of the flux auto-correlation function. More specifically, two dividing surfaces are employed and placed at the top of each barrier and two correlation functions are introduced: $C_{R, R}(t)$ when both dividing surfaces are located at the top of the first barrier (the reactants side) and $C_{R, P}(t)$ when the dividing surfaces are located at each barrier top (reactant and product side respectively). Since Eq. (1) is exact, each correlation function gives the exact thermal rate and, in particular, any weighted linear combination of them will as well. Peskin [29] found that a suitable combination is

$$
\begin{aligned}
k(T) Q_{R}(T)=\lim _{t \rightarrow+\infty} & \left(\frac{\left|C_{R, R}(t)\right| \int_{0}^{t} C_{R, P}\left(t^{\prime}\right) d t^{\prime}}{\left|C_{R, P}(t)\right|+\left|C_{R, R}(t)\right|}\right. \\
& \left.+\frac{\left|C_{R, P}(t)\right| \int_{0}^{t} C_{R, R}\left(t^{\prime}\right) d t^{\prime}}{\left|C_{R, P}(t)\right|+\left|C_{R, R}(t)\right|}\right)
\end{aligned}
$$

because the ratio of the two correlation functions is roughly constant after some time $t_{0}$, where $t_{0}$ denotes the end of the direct-scattering regime and the transient of the delay time regime induced by the wave-packet population barrier resonance states. Although this ap- 
proach is very powerful in many cases of interest [28-30], re-crossing phenomena can lead to a significant increase of the total computational time needed to reach convergence. Furthermore, this method usually employs imaginary absorbing potentials because the dynamics involves a long-time decay beyond the barrier region: the expression and location of such absorbing potentials is quite arbitrary and their presence can lead to fictitious effects. These shortcomings call for a better approach to tackle the calculation of the thermal rate constant. We will present our approach for this in the next Section.

\section{A TIME-INDEPENDENT APPROACH}

As described in Sec. II, time-dependent approaches become computationally demanding in the presence of many metastable states with a long lifetime. Hence, a time-independent approach is preferred for the calculation of $k(T)$ for resonating systems. In order to switch from the time-domain to the energy-domain, it is necessary to integrate out the time dependence in Eq. (1) [24]. After inserting the following identity in Eq. (2)

$$
e^{-\hat{H}(\beta / 2+i t / \hbar)}=\int_{-\infty}^{+\infty} e^{-E(\beta / 2+i t / \hbar)} \delta(E-\hat{H}) d E
$$

and integrating over time, a time-independent expression for the thermal rate constant is obtained [24]

$$
k(T) Q_{R}(T)=\frac{1}{2 \pi \hbar} \int_{-\infty}^{+\infty} e^{-\beta E} N(E) d E
$$

where

$$
N(E)=\frac{1}{2}(2 \pi \hbar)^{2} \operatorname{Tr}\left[\hat{F}_{1} \delta(E-\hat{H}) \hat{F}_{2} \delta(E-\hat{H})\right]
$$

is the cumulative reaction probability evaluated between the dividing surface $f_{1}(\mathbf{s})=0$ and $f_{2}(\mathbf{s})=0$. Eqs. (7) and (8) have been successfully used in the past for several quantum rate calculations [11, 50-55]. In particular, Manthe et al. [56] have been able to calculate the exact quantum rate for the hydrogen abstraction reaction from methane $\mathrm{H}+\mathrm{CH}_{4} \rightarrow$ $\mathrm{H}_{2}+\mathrm{CH}_{3}$. Despite their apparent simplicity, Eqs. (7) and (8) can be solved analytically only for a limited number of problems and a numerical approach is necessary otherwise. Eq. (8) closely resembles the Landauer $[57,58]$ formalism equations employed to calculate the electrical and heat current.

As described in Appendix A, the cumulative reaction probability $N(E)$ for onedimensional systems is exactly the transmission probability $T(E)$, namely the probability 
of a quantum particle to cross the scattering potential. In the following, we will describe our numerical method for computing $T(E)$ efficiently.

\section{A. The choice of boundary conditions - a fast approach time-independent problem}

The main idea of the present method consists in finding asymptotic eigenfunctions $\left|\psi_{p}\right\rangle$ of the type

$$
\hat{H}\left|\psi_{p}\right\rangle=\left[-\frac{\hbar^{2}}{2 m} \frac{d^{2}}{d x^{2}}+V(x)\right]\left|\psi_{p}\right\rangle=E\left|\psi_{p}\right\rangle
$$

using tools for solving ordinary differential equations (ODEs) at fixed eigenvalue $E$, with an appropriate choice of boundary conditions.[61] In fact, when no information about the spectrum is known, pairs of eigenvalues and eigenfunctions of Eq. (9) must be computed at the same time using standard tools for solving eigenproblems. However, when eigenvalues are known a priori (for example, in the simple case of asymptotic free systems), eigenfunctions of Eq. (9) can be directly computed solving Eq. (9) as an ODE, where the eigenvalues are treated as parameters. In this work, we consider scattering potentials with free boundary conditions (see Appendix A for details). When the potential is nonzero only in a closed region $[-L,+L]$ and zero outside, eigenfunctions are plain waves with momentum $p$ and energy $E=\frac{p^{2}}{2 m}$ in the asymptotic limit $|x| \gg L$. Because no bounded states exist for $E>0$ (in this case, all bounded states have energies $E<0$ and they exist only if $V(x)<0, x \in[-L,+L])$, we can divide the spectrum of Eq. (9) in two parts: a continuous spectrum for $E>0$ and a discrete spectrum (if present) for $E<0$. Recalling that only unbounded states are relevant for the computation of the transmission probability $T(E)$, we can focus our attention exclusively on the continuous spectrum, where the energy $E>0$ can be treated as continuous parameter in the ODE.

The last step needed to solve Eq. (9) as an ODE consists in providing an appropriate choice of the boundary conditions. Because no absorbing barriers are present, the total energy must be conserved. Therefore, the following scattering representation

$$
\psi_{p}(x)=\left\{\begin{array}{r}
\mathrm{e}^{i p x / \hbar} x \ll-L \\
\frac{t}{|t|^{2}} \mathrm{e}^{i p x / \hbar}+\frac{r}{|t|} \mathrm{e}^{-i p x / \hbar} \quad x \gg+L
\end{array}\right.
$$


can be obtained after using the following choice of boundary conditions

$$
\begin{aligned}
\psi_{p}\left(x_{0}\right) & =1 \\
\partial_{x} \psi_{p}\left(x_{0}\right) & =i p / \hbar .
\end{aligned}
$$

where $x_{0} \ll-L$. The pre-factors $\frac{t}{|t|^{2}}$ and $\frac{r}{|t|}$ in Eq. (10) are necessary in order to satisfy the continuity equation Eq. (A3). Hence, transmission and reflection probabilities become

$$
\begin{aligned}
& T(E)=|t|^{2}=4\left|\psi_{p}(\tilde{x})+\frac{\hbar}{i p} \partial_{x} \psi_{p}(\tilde{x})\right|^{-2}, \\
& R(E)=|r|^{2}=1-\left(1+\frac{1}{4}\left|\psi_{p}(\tilde{x})-\frac{\hbar}{i p} \partial_{x} \psi_{p}(\tilde{x})\right|^{2}\right)^{-1},
\end{aligned}
$$

where $\tilde{x}$ is an arbitrary point such that $\tilde{x} \gg L$. Although many other boundary conditions can be chosen, we used the boundary conditions in Eq. (11) in order to have a simple and computationally efficient expression of $T(E)$.

The method described above has many advantages. On one hand, we can find the thermal rate constant avoiding the time integration and using the transmission probability. On the other, we provide an efficient way to solve the Schrödinger equation in Eq. (9) using tools for solving ODEs provided by Mathematica suite [62] or any other equivalent package, avoiding the related eigenproblem which is numerically harder to tackle. Moreover, within the present method, the transmission probability can be sampled ad hoc, using a finer energy grid near resonant peaks. This is very important when dealing with the integration in Eq. (7) because, in the case of resonating double barrier potentials very narrow peaks are present. By using a fine grid only around resonant peaks and a coarse grid in flat regions, we have been able to reach numerically converged results for the energy integration in Eq. (7).

\section{B. Numerical method details}

For the numerical solution of the ODE Eq. (9) we used the routine NDSolve with the parameter MaxSteps $\rightarrow$ Infinity, while for the numerical evaluation of $T(E)$ we used the routine NIntegrate with the parameter WorkingPrecision $\rightarrow 15$ (WorkingPrecision $\rightarrow 50$ for the double Eckart barrier potential).[63] In our calculation we fix $T(E)=0$ for $E / \omega_{0}<10^{-4}$ and $T(E)=1$ for $E / \omega_{0}>10^{-1}\left(E / \omega_{0}>3 \cdot 10^{-1}\right.$ for the double square barrier potential), 
TABLE I: Interval of energies $\left[E_{\min }, E_{\max }\right]$ and energy step $\Delta E$ used for computing the transmission probability $T(E)$ for each potential $\left(\omega_{0}=27.211 \mathrm{eV}\right)$.

\begin{tabular}{|c|c|c|c|}
\hline Potential & $E_{\min } / \omega_{0}$ & $\mathrm{E}_{\max } / \omega_{0}$ & $\Delta E / \omega_{0}$ \\
\hline Single Eckart barrier (Fig. 1) & $10^{-4}$ & $10^{-1}$ & $10^{-4}$ \\
\hline Double rectangular barrier (Fig. 2) & $10^{-4}$ & $3 \cdot 10^{-1}$ & $10^{-4}$ \\
\hline \multirow{3}{*}{ Single resonant peak, Eq. (13) (Fig. 3) } & $5 \cdot 10^{-4}$ & $2 \cdot 10^{-2}$ & $5 \cdot 10^{-4}$ \\
\hline & $5 \cdot 10^{-3}$ & $6 \cdot 10^{-3}$ & $10^{-5}$ \\
\hline & $2 \cdot 10^{-2}$ & $10^{-1}$ & $10^{-3}$ \\
\hline \multirow{6}{*}{ Double Eckart barrier (Fig. 5) } & $10^{-4}$ & $5 \cdot 10^{-2}$ & $10^{-4}$ \\
\hline & $1.08 \cdot 10^{-3}$ & $1.10 \cdot 10^{-3}$ & $10^{-6}$ \\
\hline & $3.23 \cdot 10^{-3}$ & $3.25 \cdot 10^{-3}$ & $10^{-6}$ \\
\hline & $5.34 \cdot 10^{-3}$ & $5.36 \cdot 10^{-3}$ & $10^{-6}$ \\
\hline & $7.39 \cdot 10^{-3}$ & $7.41 \cdot 10^{-3}$ & $10^{-6}$ \\
\hline & $9.31 \cdot 10^{-3}$ & $9.33 \cdot 10^{-3}$ & $10^{-6}$ \\
\hline
\end{tabular}

where $\omega_{0}=27.211 \mathrm{eV}$ is the conversion factor between the atomic units of energy and $\mathrm{eV}$. In Tab. (I), the dimensions of the grids for each potential are reported.

\section{RESULTS}

The typical textbook example for testing tunneling rate methods is the Eckart potential. A particle of $m=1.060 \times 10^{3}$ a.u. is scattered against an Eckart barrier $V(x)=V_{0} / \cosh ^{2}(\pi x / a)$, where $V_{0}=0.424 \mathrm{eV}$ and $a=2.305$ a.u. The potential profile is reported in the top panel in Fig. (1). In the same figure, the central panel shows the transmission probability $T(E)$. In order to better appreciate the difference between the exact (continuous line) and the calculated (dots) values, the relative error is reported in the inset. This shows that the percentage error is always smaller than $0.03 \%$. The values of $k(T) \cdot Q_{R}$ are reported in the bottom panel and there is almost no deviation from the analytical results even at very low temperatures. For example at $T=60 \mathrm{~K}$ the percentage 

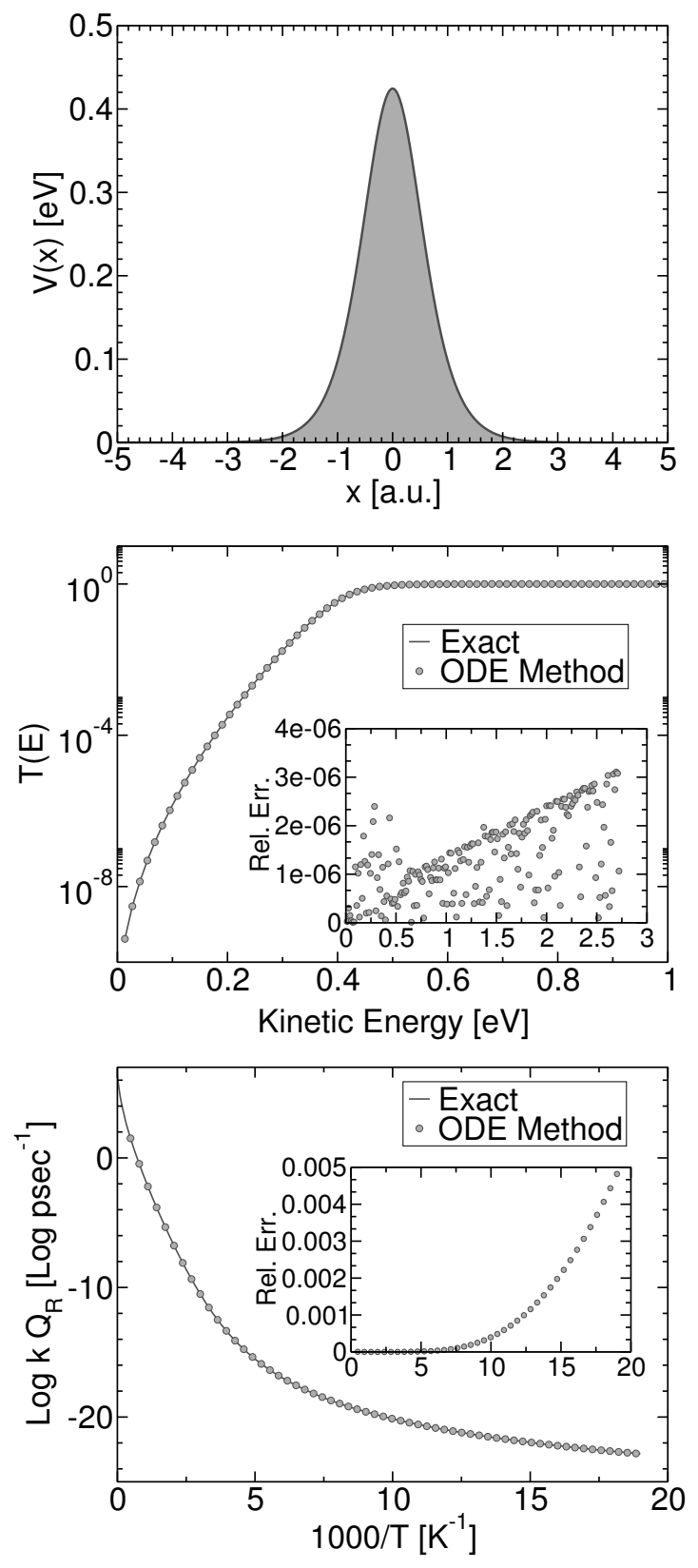

FIG. 1: Thermal rate constant for a single Eckart barrier potential. (Top panel) The potential profile. (Middle panel) Comparison between the exact (solid) transmission probability $T(E)$ and our numerical results using the ODE method (dots). As reported in the inset, the relative error $\left|\frac{T_{\mathrm{ODE}}(E)-T_{\text {exact }}(E)}{T_{\text {exact }}(E)}\right|$ is smaller than $3 \times 10^{-6}$. (Lower panel) Comparison between the exact (solid) thermal rate constant $k Q_{r}$ and our numerical results using the ODE method (dots). In the inset, the relative error $\left|\frac{\left(k Q_{R}\right)_{\mathrm{ODE}}-\left(k Q_{R}\right)_{\text {exact }}}{\left(k Q_{R}\right)_{\text {exact }}}\right|$ is shown. 
error is smaller than half a percent.

The main goal of this paper is to look at resonance barrier scattering rate constants. In order to test the method in the case of an analytical example, we have chosen the double rectangular barrier, whose profile is reported in the top panel of Fig. (2). The particle's mass and the potential maximum value are the same as for the Eckart barrier calculation. The rectangular barriers width has been set equal to $\Delta=0.25$ a.u. and the gap between the barriers equal to 1 a.u.. As shown in the middle panel of Fig. (2), three quasi-bound states can clearly be distinguished from the $T(E)$ profile. Despite the discontinuity of the first derivative of the double rectangular barrier potential, the numerical solution is in accordance with the analytical result for the entire energy range, as in the case of the Eckart potential. The same considerations can be made for the bottom panel, where the thermal rate constant is reported.

As a third example, a well-known resonating potential has been chosen from the literature. [29] This potential is made of two Eckart potentials and it has been employed to test other methods for the calculation of thermal rate constants of resonating systems, such as the FAM recalled above. Following Peskin et al. [29], the mass of the scattering particle is set to $m=1.834 \times 10^{3}$ a.u. and the potential energy function is

$$
V(x)=V_{0}\left(\frac{1}{\cosh ^{2}(x)}-\frac{1}{\cosh ^{2}(a x)}\right),
$$

where $V_{0}=0.310 \mathrm{eV}$ and $a=5 \mathrm{a} . \mathrm{u}$. The potential profile is reported in the top panel of Fig. (3). The potential gap is such that there is a single metastable state, as shown in the middle panel where there is a single transmission probability resonance peak. To use the FAM formulation of the thermal rate of Eq. (5), we calculate the flux auto-correlation functions $C_{R, R}(t)$ and $C_{R, P}(t)$ using a sinc-DVR grid method [64]. As an example, the value and the profile of Eq. (5) versus the truncation times $t_{0}$ at $T=250 \mathrm{~K}$ are reported in Fig. (4). These are in close agreement with the time-independent method presented here and reported on the same figure as a horizontal dashed line. The complete set of thermal rate constant results are reported on the bottom panel of Fig. (3). In particular, the inset reveals that the agreement between the FAM method and the present is within $4 \%$ down to $50 \mathrm{~K}$.

Finally an even more challenging test has been performed. This is represented by the potential in Fig. (5), where two Eckart barriers with a gap such that several quasi-bound 

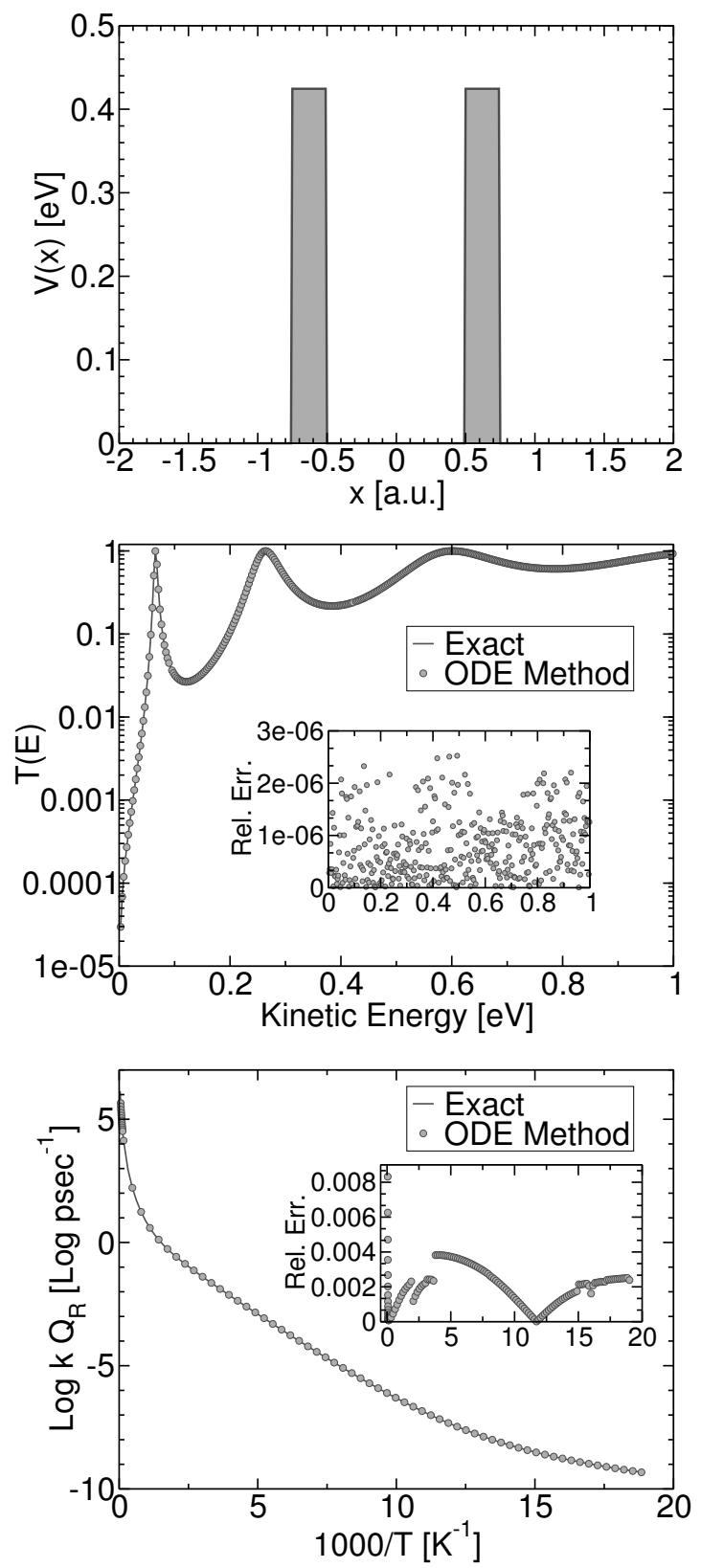

FIG. 2: Thermal rate constant for a double rectangular barrier potential. (Top panel) The potential profile. (Middle panel) Comparison between the exact (solid) transmission probability $T(E)$ and our numerical results using the ODE method (dots). As reported in the inset, the relative error $\left|\frac{T_{\mathrm{ODE}}(E)-T_{\text {exact }}(E)}{T_{\text {exact }}(E)}\right|$ is smaller than $3 \times 10^{-6}$. (Lower panel) Comparison between the exact (solid) thermal rate constant $k Q_{r}$ and our numerical results using the ODE method (dots). In the inset, the relative error $\left|\frac{\left(k Q_{R}\right)_{\mathrm{ODE}}-\left(k Q_{R}\right)_{\text {exact }}}{\left(k Q_{R}\right)_{\text {exact }}}\right|$ is shown. 

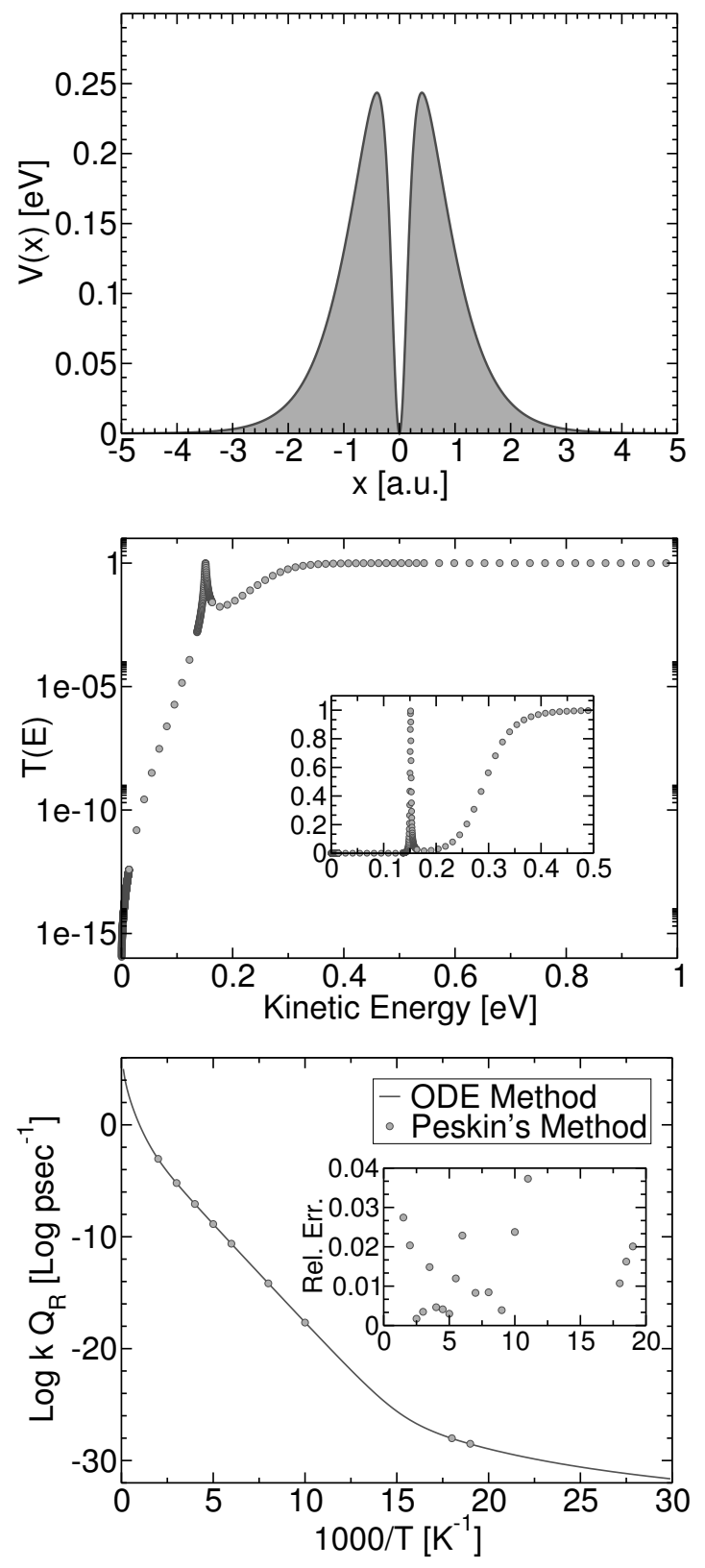

FIG. 3: Thermal rate constant for a double barrier potential, Eq. (13). (Top panel) The potential profile. (Middle panel) Transmission probability $T(E)$ calculated using the ODE method (log-lin scale for the main figure and lin-lin scale for the inset). (Lower panel) Comparison between the thermal rate constant $k Q_{r}$ computed using our numerical method (solid) and the FAM method (dots). In the inset, the relative error $\left|\frac{\left(k Q_{R}\right)_{\mathrm{ODE}}-\left(k Q_{R}\right)_{\mathrm{FAM}}}{\left(k Q_{R}\right)_{\mathrm{FAM}}}\right|$ is shown. The deviation between the two numerical methods is contained within $4 \%$. 


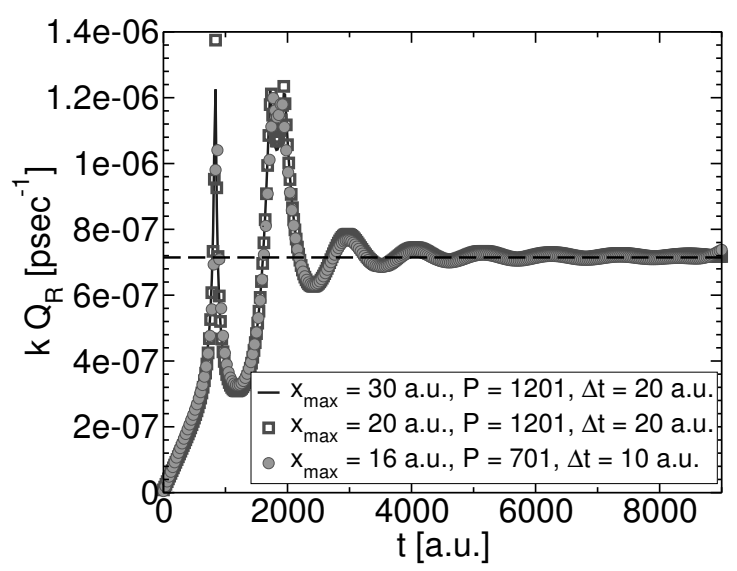

FIG. 4: Thermal rate constant as a function of the truncation time $t_{0}$ obtained using the FAM method of Eq. (5) at $T=125 \mathrm{~K}$ and for the potential of Eq. (13). The dotted line is the value of $k Q_{r}$ obtained using our ODE method. $x_{\max }$ denotes the DVR grid extension, $P$ the number of points and $\Delta t$ the time increment in the time-dependent simulation.

states are generated. The particle's mass is the same for the potential in Eq. (13) and the potential equation is

$$
V(x)=V_{0}\left(\frac{1}{\cosh ^{2}(x-a)}+\frac{1}{\cosh ^{2}(x+a)}-\frac{2}{\cosh ^{2}(a) \cosh ^{2}(x)}\right)
$$

where $V_{0}=0.310 \mathrm{eV}$ and $a=2 \mathrm{a} . \mathrm{u}$. Also in this case, we set $m=1.834 \times 10^{3}$. The potential profile is shown in the top panel of Fig. (5) and the transmission probability in the middle one. This potential represents a real challenge for a numerical method for calculating thermal rate constants, because several resonance peaks are present in the transmission probability and a wider logarithmic range has to be taken in order to describe all resonating rates. In the inset of the middle panel plot of Fig. (5) the transmission probability is reported in linear scale. With this plotting choice, resonant peaks are even more evident. When carrying out the energy integration of Eq. (7) for such a severe resonant system, one needs to be very careful in choosing the integration grid set-up around the resonance peaks. With the method labeled "ODE method" in Fig. (5) the grid density has been properly enhanced around the resonance peaks. With the method labeled "ODE method + BW Correction", a Breit-Wigner distribution is used to fit the resonance peaks of the transmission probability, in order to have a better integration of $T(E)$ and overcome the numerical limitation of having a very dense grid near peaks. More specifically, the narrow peaks are well approximated by the 

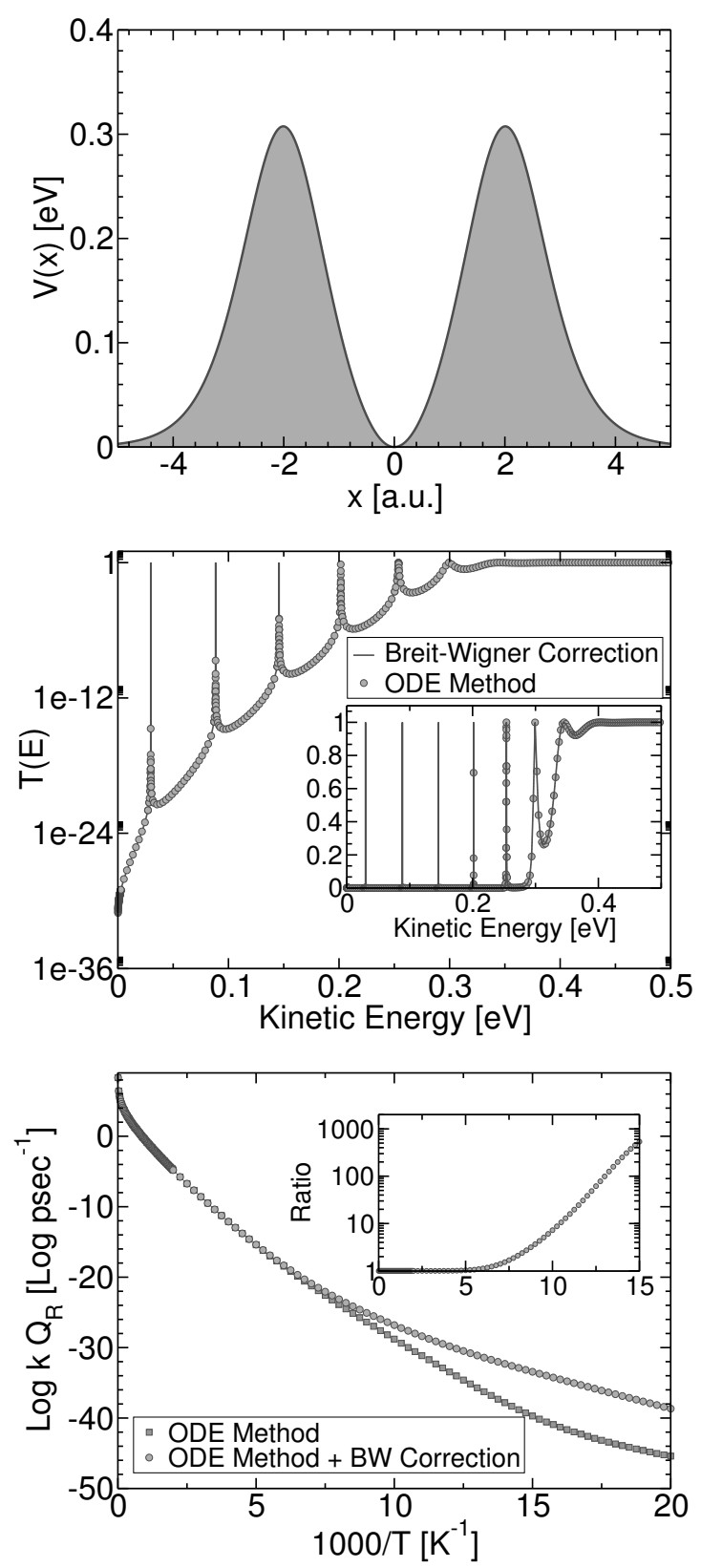

FIG. 5: Thermal rate constant for a double Eckart barrier potential, Eq. (14). (Top panel) The potential profile. (Middle panel) Transmission probability $T(E)$ computed using our ODE method with (solid) and without (dots) the Breit-Wigner correction (log-lin scale for the main figure and lin-lin scale for the inset). (Lower panel) Comparison between thermal rate constants computed using the ODE method with (circles) and without (squares) the Breit-Wigner correction. In the inset, the ratio $\left|\frac{\left(k Q_{R}\right)_{\mathrm{ODE}+\mathrm{BW}}}{\left(k Q_{R}\right)_{\mathrm{ODE}}}\right|$ is shown. 
TABLE II: Breit-Wigner distribution parameters for Eq. 15 employed for the double Eckart potential rate calculation $\left(\omega_{0}=27.211 \mathrm{eV}\right)$.

\begin{tabular}{ccc}
\hline Peak & $E_{r} / \omega_{0}$ & $\Gamma_{r} / \omega_{0}$ \\
\hline I & $1.090 \cdot 10^{-3}$ & $2.936 \cdot 10^{-15}$ \\
\hline II & $3.247 \cdot 10^{-3}$ & $9.540 \cdot 10^{-12}$ \\
\hline III & $5.358 \cdot 10^{-3}$ & $3.280 \cdot 10^{-9}$ \\
\hline IV & $7.408 \cdot 10^{-3}$ & $2.664 \cdot 10^{-7}$ \\
\hline V & $9.326 \cdot 10^{-3}$ & $1.648 \cdot 10^{-5}$ \\
\hline
\end{tabular}

Breit-Wigner (BW) approximation

$$
T_{r}(E) \approx \frac{\left(\Gamma_{r} / 2\right)^{2}}{\left(E-E_{r}\right)^{2}+\left(\Gamma_{r} / 2\right)^{2}},
$$

where $E_{r}$ and $\Gamma_{r}$ are respectively the resonance energy and the width of the resonant peak. A JWKB (Jeffreys-Wenzel-Brillouin-Kramer) [65] derivation of the BW distribution for these resonance peaks is given in Appendix B. Consequently, we corrected our "ODE method" using an analytical expression for the transmission probability at the center of the narrowest peaks. Differences between the two approaches can be appreciated only at very low temperatures, namely below $T=200 \mathrm{~K}$. At these temperatures, the BW fitting resulted to be necessary in order to have an accurate thermal rate constant value. In Tab. (II), we report values of $E_{r}$ and $\Gamma_{r}$ (starting from the lowest in energy peak) obtained fitting Eq. (15) near each peak. 


\section{IMPORTANCE OF THE RESONANT PEAKS FOR THE CALCULATION OF THE THERMAL RATE CONSTANT $k(T)$ IN THE DEEP RESONANT TUNNEL- ING REGIME}

In general, the thermal rate constant $k(T)$ in Eq. (7) can be written as the sum of a singular part (resonant contribution) and a non-singular part

$$
\begin{aligned}
k(T) Q_{R}(T) & =\frac{1}{2 \pi \hbar} \sum_{i=1}^{n_{p}} \int_{E_{i}-\Delta}^{E_{i}+\Delta} e^{-\beta E} N_{i}(E) d E \\
& +\frac{1}{2 \pi \hbar} \int_{0}^{+\infty} e^{-\beta E} N_{\mathrm{NR}}(E) d E
\end{aligned}
$$

where $n_{p}$ is the number of resonant peaks. In Eqs. (16), $N_{i}(E)$ and $N_{\mathrm{NR}}(E)$ are respectively the cumulative transmission probabilities in proximity of the $i-t h$ resonant peak and outside singular regions, and $\Delta$ is supposed to be small enough to allow for a clear separation of each resonance peak. In the case of symmetric double barriers, the resonance transmission probability of each resonance peak $N_{i}(E)$ is well approximated by Eq. (15) as shown in Appendix B. For sufficiently small width $\Gamma_{i}, N_{i}(E)$ can be further approximated, for analytical integration purposes, by [27]

$$
N_{i}(E)=\frac{\left(\Gamma_{i} / 2\right)^{2}}{\left(E-E_{i}\right)^{2}+\left(\Gamma_{i} / 2\right)^{2}} \approx \frac{\pi \Gamma_{i}}{2} \delta\left(E-E_{i}\right) .
$$

Using Eq. (17), we obtain an estimate of the resonant contribution to the thermal rate constant as

$$
k_{\mathrm{res}}(T) Q_{R}(T) \approx \frac{1}{2 \pi \hbar} \frac{\pi}{2} \sum_{i=1}^{N_{P}} \Gamma_{i} e^{-\beta E_{i}},
$$

which depends directly on each finite width $\Gamma_{i}$ and resonant energy $E_{i}$.

Because the resonant part in Eq. (18) is a sum of exponentials, $k_{\text {res }}(T) Q_{R}(T)$ will be dominated by those peaks $i^{*}$ for which $E_{i^{*}} \approx \frac{1}{\beta}=k_{B} T$ and then

$$
\log \left[k(T) Q_{R}(T)\right] \approx-\beta E_{i^{*}}+A(\beta)
$$

where $A(\beta)$ is a slowly changing function which contains the contribution of the non-resonant part in Eq. (16b). Therefore, as a consequence of the resonant tunneling, the interplay between the resonant peak weights induces, as the temperature is lowered, a clear variation of the slope in a log-lin plot of the thermal rate constant $k(T)$. It is important to observe that 
this phenomenon is completely absent in non-resonant potentials where in deep tunneling regime the logarithm of the thermal rate is constant.

For this reason, it is necessary to include all resonant peaks in the transmission probability integration when resonant potentials are considered. In this direction, we performed some numerical tests by artificially removing the lower energy peaks and we have found that the thermal rate to change drastically (see for instance Fig. 5), even if the Boltzmann weight reduces the contribution coming from the lower energy peaks.

\section{CONCLUSIONS}

This paper presents a method for calculating thermal rate constants $k(T)$ for resonating one dimensional scattering potentials in the presence of energy barriers. After showing that thermal rate constants can be calculated from asymptotic conditions, the Schrödinger equation has been solved as an ordinary differential equation, with the energy as a fixed parameter, by choosing convenient boundaries conditions. The method we propose is time-independent which provides a significant advantage over any available time-dependent method for calculating rate constants in the presence of resonant states. We have shown this by calculating $k(T)$ for arbitrary potentials, even for first-derivative discontinuous potentials as well as the double barrier potential with several metastable states. In both cases, the error respect to the exact expression was less than $5 \%$ even at extremely low temperatures. Possible multidimensional implementations of the method are under way in our group.

\section{Acknowledgments}

Prof. E. Pollak and Prof. J. Schrier are thanked for useful discussions. The University of Milan is thanked for funding (PUR grant) and CILEA (Consorzio Interuniversitario Lombardo per L'Elaborazione Automatica) for computational time allocation.

\section{Appendix A: Cumulative reaction probability and probability current}

In this section we provide a useful expression for the cumulative reaction probability $T(E)$ in terms of probability currents. We take advantage of the fact that, in the asymptotic limit 
and for one dimensional scattering potentials, probability currents are related in a simple manner to eigenfunctions. Let $\left|\psi_{p}\right\rangle$ be the set of eigenfunctions far from the scattering interaction region and $p$ the associated momentum eigenvalue. After inserting the resolution of identity $\left|\psi_{p}\right\rangle\left\langle\psi_{p}\right|$, Eq. (8) becomes

$$
\begin{aligned}
N(E) & =\frac{1}{2}(2 \pi \hbar)^{2} \iint d p d p^{\prime} \delta(E(p)-E) \delta\left(E\left(p^{\prime}\right)-E\right) \\
& \times\left\langle\psi_{p}\left|\hat{F}_{1}\right| \psi_{p^{\prime}}\right\rangle\left\langle\psi_{p^{\prime}}\left|\hat{F}_{2}\right| \psi_{p}\right\rangle .
\end{aligned}
$$

where the integration over the momenta $p$ and $p^{\prime}$ denotes the sum over all eigenfunctions [59]. Using the definition of the flux operators in Eq. (3), the flux matrix elements become

$$
\begin{aligned}
\left\langle\psi_{p}\left|\hat{F}_{1}\right| \psi_{p^{\prime}}\right\rangle & =\frac{d}{d t} \int_{-\infty}^{+\infty} d \mathbf{s} \psi_{p}^{\star}(\mathbf{s}) \hat{h}\left(f_{1}(\mathbf{s})\right) \psi_{p^{\prime}}(\mathbf{s}) \\
& =\int_{f_{1}(\mathbf{s}) \geqslant 0} d \mathbf{s} \frac{d}{d t}\left(\psi_{p}^{\star}(\mathbf{s}) \psi_{p^{\prime}}(\mathbf{s})\right)
\end{aligned}
$$

where the integration in Eq. (A2b) is limited to the regions where $f_{1}(\mathbf{s}) \geqslant 0$ (products region). Using the continuity equation

$$
\frac{d}{d t}\left(\psi_{p}^{\star}(\mathbf{s}) \psi_{p^{\prime}}(\mathbf{s})\right)=\vec{\nabla}_{\mathbf{s}} \cdot \vec{j}_{p p^{\prime}}(\mathbf{s}),
$$

where

$$
\vec{j}_{p p^{\prime}}(\mathbf{s})=-\frac{i \hbar}{2 m}\left[\psi_{p}^{*}(\mathbf{s}) \vec{\nabla}_{\mathbf{s}} \psi_{p^{\prime}}(\mathbf{s})-\vec{\nabla}_{\mathbf{s}} \psi_{p}^{\star}(\mathbf{s}) \psi_{p^{\prime}}(\mathbf{s})\right]
$$

is the probability current, and then, applying the "Divergence Theorem", the expression of the flux matrix elements becomes

$$
\left\langle\psi_{p}\left|\hat{F}_{1}\right| \psi_{p^{\prime}}\right\rangle=\int_{f_{1}(\mathbf{s})=0} d \mathbf{s} \vec{j}_{p p^{\prime}}(\mathbf{s}) \cdot \overrightarrow{\mathbf{n}}_{1}
$$

where $\overrightarrow{\mathbf{n}}_{1}$ is a unit vector normal to the dividing surface $f_{1}(\mathbf{s})=0$. After substituting Eq. (A5) into Eq. (A1) for both flux operators, the expression of the cumulative reaction probability in terms of the probability current becomes

$$
\begin{aligned}
N(E) & =\frac{1}{2}(2 \pi \hbar)^{2} \iint d p d p^{\prime} \delta(E(p)-E) \delta\left(E\left(p^{\prime}\right)-E\right) \\
& \times\left(\int_{f_{1}(\mathbf{s})=0} d \mathbf{s} \vec{j}_{p p^{\prime}}(\mathbf{s}) \cdot \overrightarrow{\mathbf{n}}_{1}\right)\left(\int_{f_{2}\left(\mathbf{s}^{\prime}\right)=0} d \mathbf{s}^{\prime} \vec{j}_{p p^{\prime}}\left(\mathbf{s}^{\prime}\right) \cdot \overrightarrow{\mathbf{n}}_{2}\right)^{\star} .
\end{aligned}
$$

In this paper resonant rate calculations is carried out for arbitrary one dimensional scattering potentials $V(x)$, with the asymptotic condition $V(x)=0$ for $|x| \gg L$ and the interval 
$[-L,+L]$ delimits the nonzero potential region. Therefore, we can assume that the dividing surface equation is of the form $f(x)=x-x_{0}=0$, where $x_{0}$ is the position of the dividing surface. As introduced in Section II, a convenient choice of the flux operators has often been to place them one in the reactants and the other in the products side, in particular for resonant scattering. Here, we choose to place them in the same asymptotic location $f_{1}(x)=f_{2}(x)=x-x_{0}$, where $x_{0}$ is either $x_{0} \ll-L$ or $x_{0} \gg+L$. It is important to remember that the actual rate constant is independent of the location of the dividing surfaces, but appropriate choices of the dividing surfaces locations may reduce the computational effort required to evaluate the thermal rate constant. Given this choice, after integrating at the dividing surface point, Eq. (A6) becomes

$$
\begin{aligned}
N(E) & =\frac{1}{2}(2 \pi \hbar)^{2} \iint d p d p^{\prime} \delta(E(p)-E) \\
& \times \delta\left(E\left(p^{\prime}\right)-E\right)\left|j_{p p^{\prime}}\left(x_{0}\right)\right|^{2}
\end{aligned}
$$

where the cumulative reaction probability can be clearly interpreted as a counter of the number of particles that cross the dividing surface.

In order to calculate $T(E)$ from asymptotic conditions, one needs the expression of the eigenfunctions $\left|\psi_{p}\right\rangle$ in these regions. When a wave is incoming (left to right), the eigenfunctions are

$$
\psi_{p}^{R}(x)=\left\{\begin{aligned}
\frac{1}{\sqrt{2 \pi \hbar}}\left(\mathrm{e}^{i p x / \hbar}+r \mathrm{e}^{-i p x / \hbar}\right) & x \ll-L \\
\frac{1}{\sqrt{2 \pi \hbar}} t \mathrm{e}^{i p x / \hbar} & x \gg+L
\end{aligned}\right.
$$

and when it is outgoing (right to left) they are

$$
\psi_{p}^{L}(x)=\left\{\begin{aligned}
\frac{1}{\sqrt{2 \pi \hbar}} t \mathrm{e}^{-i p x / \hbar} & x \ll-L \\
\frac{1}{\sqrt{2 \pi \hbar}}\left(\mathrm{e}^{-i p x / \hbar}+r \mathrm{e}^{i p x / \hbar}\right) & x \gg+L
\end{aligned}\right.
$$

where the energy for both eigenfunctions is set to $E=p^{2} / 2 m$ and no absorbing potential is employed. Depending of the choice of the dividing surface, one will use Eqs. (A8) for the case of $x_{0} \gg+L$ or $x_{0} \ll-L$. Recalling that $T=|t|^{2}$ and $R=|r|^{2}$ represent respectively the transmission and reflection coefficients with $R+T=1$, it can be shown that for both 
asymptotic choices of $x_{0}$, the values of the probability currents are

$$
\begin{aligned}
j_{p p}^{R R}\left(s_{0}\right) & =\frac{T}{\pi \hbar} \sqrt{\frac{E}{2 m}} \\
j_{p p}^{L L}\left(s_{0}\right) & =\frac{R-1}{\pi \hbar} \sqrt{\frac{E}{2 m}} \\
j_{p p}^{R L}\left(s_{0}\right)=j_{p p}^{L R}\left(s_{0}\right)^{*} & =\frac{r t^{*}}{\pi \hbar} \sqrt{\frac{E}{2 m}},
\end{aligned}
$$

where the steady state flux conservation relation $t^{*} r+r^{*} t=0$ is used to show that Eqs. (A9) are the same for the case of $x_{0} \gg+L$ and $x_{0} \ll-L$. Taking into account that the overall probability current is the sum over all possible probability currents (right to right, right to left, etc.) [60], Eq. (A7) becomes

$$
\begin{aligned}
N(E) & =\frac{1}{2}(2 \pi \hbar)^{2} \rho(E)^{2}\left(\left|j_{p p}^{R R}\left(s_{0}\right)\right|^{2}+\left|j_{p p}^{L L}\left(s_{0}\right)\right|^{2}+2\left|j_{p p}^{L R}\left(s_{0}\right)\right|^{2}\right) \\
& =\frac{1}{2}(2 \pi \hbar)^{2} \rho(E)^{2} \frac{E}{m \pi^{2} \hbar^{2}} T(E)
\end{aligned}
$$

where $\rho(E)$ is the density of states for $x_{0} \gg+L$ or $x_{0} \ll-L$ and where we explicitly indicate the energy dependence of the transmission coefficient $T(E)$. [57] For one dimensional systems, the free-particle density of states is $\rho(E)=\sqrt{m / 2 E}$ and then the cumulative reaction probability $N(E)$ results to be exactly the transmission probability $T(E)$.

\section{Appendix B: The WKB Approximation for the Double Barrier}

The WKB wave functions before, between and after the barriers reported in Fig. 6 are respectively

$$
\begin{aligned}
\psi_{I}(x) & =\frac{A}{\sqrt{k(x)}} e^{i\left(\frac{\pi}{4}+\int_{x_{1}}^{x} k\left(x^{\prime}\right) d x^{\prime}\right)}+\frac{B}{\sqrt{k(x)}} e^{-i\left(\frac{\pi}{4}+\int_{x_{1}}^{x} k\left(x^{\prime}\right) d x^{\prime}\right)} \\
\psi_{I I I}(x) & =\frac{C}{\sqrt{k(x)}} e^{i\left(\frac{\pi}{4}+\int_{x_{2}}^{x} k\left(x^{\prime}\right) d x^{\prime}\right)}+\frac{D}{\sqrt{k(x)}} e^{-i\left(\frac{\pi}{4}+\int_{x_{2}}^{x} k\left(x^{\prime}\right) d x^{\prime}\right)} \\
\psi_{V}(x) & =\frac{E}{\sqrt{k(x)}} e^{i\left(\frac{\pi}{4}+\int_{x_{4}}^{x} k\left(x^{\prime}\right) d x^{\prime}\right)}+\frac{F}{\sqrt{k(x)}} e^{-i\left(\frac{\pi}{4}+\int_{x_{4}}^{x} k\left(x^{\prime}\right) d x^{\prime}\right)},
\end{aligned}
$$

where $k(x)$ is the one dimensional wave vector, $x_{i}$ the $i$-th turning point, and the coefficients are reported in Fig. (6). After applying the barrier connection formula twice [67], the coefficients $E$ and $F$ in terms of $A$ and $B$ are

$$
\left(\begin{array}{l}
E \\
F
\end{array}\right)=\left(\begin{array}{cc}
\sqrt{1+e^{2 \theta_{2}}}, & -e^{\theta_{2}} \\
-e^{\theta_{2}} & \sqrt{1+e^{2 \theta_{2}}}
\end{array}\right)\left(\begin{array}{c}
\left(\sqrt{1+e^{2 \theta_{1}}} A-B e^{\theta_{1}}\right) e^{i \phi} \\
\left(\sqrt{1+e^{2 \theta_{1}}} B-A e^{\theta_{1}}\right) e^{-i \phi}
\end{array}\right),
$$




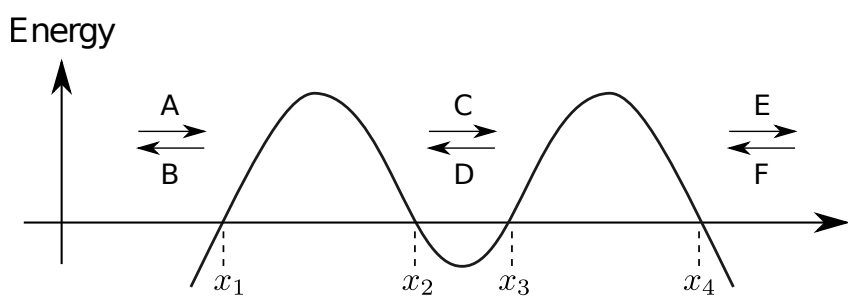

FIG. 6: Double barrier potential and turning points: A, B, C, D, E, and F are the WKB wavefunction coefficients in the three allowed regions.

where

$$
\begin{aligned}
\theta_{1} & =\int_{x_{1}}^{x_{2}}|k(x)| d x \\
\theta_{2} & =\int_{x_{3}}^{x_{4}}|k(x)| d x \\
\phi & =\int_{x_{2}}^{x_{3}} k(x) d x .
\end{aligned}
$$

In order to calculate the transmission probability of an incoming wave from the left, the coefficient $F$ is set to zero and $B$ is consequently expressed in terms of $A$. Then, the ratio is

$$
\frac{E}{A}=\frac{1}{e^{\theta_{1}} e^{\theta_{2}} e^{i \phi}+\sqrt{1+e^{2 \theta_{1}}} \sqrt{1+e^{2 \theta_{2}}} e^{-i \phi}}
$$

and the transmission probability is the modulus square of Eq. (B4) [68]

$$
T(E)=\frac{p_{1} p_{2}}{1+\left(1-p_{1}\right)\left(1-p_{2}\right)+2 \sqrt{1-p_{1}} \sqrt{1-p_{2}} \cos (2 \phi)},
$$

where

$$
p_{i}=\frac{1}{1+e^{\theta_{i}}}, \quad i=1,2 .
$$

Taking a symmetric double barrier $\left(p_{1}=p_{2}=p\right)$ and expanding $\phi(E) \approx \phi\left(E_{i}\right)+$ $\phi^{\prime}\left(E_{i}\right)\left(E-E_{i}\right)$ at the $i-t h$ resonant energy, Eq. (B5) is approximated as

$$
T(E) \approx \frac{\left(\Gamma_{i} / 2\right)^{2}}{\left(E-E_{i}\right)^{2}+\left(\Gamma_{i} / 2\right)^{2}},
$$

where the Breit-Wigner distribution width is

$$
\Gamma_{i}=\frac{e^{-2 \theta_{i}}}{\phi^{\prime}\left(E_{i}\right) \sqrt{1-e^{-2 \theta_{i}}}} \approx \frac{e^{-2 \theta_{i}}}{\phi^{\prime}\left(E_{i}\right)} .
$$

[1] E. Wigner, Trans. Faraday Soc. 1938, 34, 29. 
[2] H. Eyring, Chem. Rev. 1935, 17, 65; H. Eyring, J. Chem. Phys. 1935, 3, 107.

[3] M. G. Evans and M. Polanyi, Trans. Faraday Soc. 1935, 31, 875.

[4] L. L. Chang, L. Esaki, and R. Tsu, Appl. Phys. Lett. 1974, 24, 593.

[5] M. Hirose, M. Morita, and Y. Osaka, Jpn. J. Appl. Phys. 1977, 16, 561.

[6] G. Bastard, J.A. Brum, and R. Ferreira, Solid State Phys. 1991, 44, 229; R. Ferreira and G. Bastard, Rep. Prog. Phys. 1997, 60, 345.

[7] R. Schuster et al., Nature (London) 1997, 385, 417.

[8] A. Yariv, Optical Electronics, 4th ed. (Saunders), Philadelphia, (1991).

[9] J. D. Gezelter and W. H. Miller, J. Chem. Phys. 1995, 103, 7868.

[10] T. C. Germann and W. H. Miller, J. Phys. Chem. A 1997, 101, 6358.

[11] A. Viel, C. Leforestier, and W. H. Miller, J. Chem. Phys. 1998, 108, 3489.

[12] T. C. Germann and W. H. Miller, J. Chem. Phys. 1998, 109, 94.

[13] B. Ricco and M. Ya. Azbel, Phys. Rev. B 1984, 29, 1970.

[14] E. Pollak and R. Naaman, Chem. Phys. Lett. 1986, 123, 352.

[15] V. Engel, R. Schinke and E. Pollak, J. Chem. Phys. 1987, 87, 1596.

[16] J. Dai and J. Z. H. Zhang,J. Phys. Chem. 1996, 100, 6898

[17] S. Y. Lin, Z. Sun, H. Guo, D. H. Zhang, P. Honvault, D. Xie, and S.-Y. Lee, J. Phys. Chem. A $2008, \mathbf{1 1 2}, 602$

[18] H. S. Johnston, "Gas Phase reaction rate theory", Ronald Press Co., New York, (1966)

[19] E.O. Kane, "Basic concepts of tunneling" in Tunneling phenomena in solids. Plenum; 1969. p. $1-11$

[20] Y. Zohta, Phys. Rev. B 1990, 41, 7879.

[21] R. Tsu and L. Esaki, Appl. Phys. Lett. 1973, 22, 562.

[22] A. Dutt and S. Kar, Am. J. Phys. 2010, 78, 1352.

[23] U. Peskin, N. Moiseyev, and R. Lefebvre, J. Chem. Phys. 1990, 92, 2902; E. Narevicius and N. Moiseyev, J. Chem. Phys. 2000, 113, 6088; H. Barkay and N. Moiseyev, Phys. Rev. A 2001, 64, 044702; H. Barkay, E. Narevicius, and N. Moiseyev, Phys. Rev. B 2003, 67, 045322; T. Goldzak, I. Gilary, and N. Moiseyev, Phys. Reb. A 2010, 82, 052105.

[24] W. H. Miller, S. D. Schwartz, and J. W. Tromp, J. Chem. Phys. 1983, 79, 4889.

[25] W. H. Miller, J. Phys. Chem. A 1998, 102, 793.

[26] T. Yamamoto and W. H. Miller, J. Chem. Phys. 2003, 118, 2135. 
[27] V. Ryaboy and R. Lefebvre, J. Chem. Phys. 1993, 99, 9547.

[28] L. Berman and U. Peskin, Chem. Phys. Lett. 2002, 360, 374.

[29] M Caspary, L. Berman, and U. Peskin, Chem. Phys. Lett. 2003, 369, 232.

[30] L. Berman and U. Peskin, Int. J. Quantum Chem. 2004, 99, 752; M. Caspary Toroker and U. Peskin, Chem. Phys. 2010, 370, 124.

[31] M. Topaler and N. Makri, J. Chem. Phys. 1994, 101, 7500; N. J. Wright and N. Makri, J. Chem. Phys. 2003, 119, 1634; M. Topaler and N. Makri, Chem. Phys. Lett. 1993, 210, 285.

[32] J. Liu and W. H. Miller, J. Chem. Phys. 2007, 127, 114506.

[33] M. Thoss, H. Wang and W. H. Miller, J. Chem. Phys. 2001, 115, 2991; H. Wang, M. Thoss, K.- L. Sorge, R. Gelabert, X. Gimenez, and W. H. Miller, J. Chem. Phys. 2001, 114, 2562; H. Wang, M. Thoss, and W. H. Miller, J. Chem. Phys. 2000, 112, 47.

[34] T. F. Miller III and D. Manolopoulos, J. Chem. Phys. 2005, 122, 184503.

[35] E. Pollak, J. Chem. Phys. 2007, 127, 074505.

[36] H. Li, J. A. Poulsen, and G. Nyman, J. Phys. Chem. A 2011, 115, 7338; W. H. Miller, Faraday Discu. 1998, 110, 1.

[37] N. Makri, Annu. Rev. Phys. Chem. 1999, 50, 167; N. Nakayama and N. Makri, J. Chem. Phys. 2003, 119, 8592 .

[38] J. Liu and W. H. Miller, J. Chem. Phys. 2007, 126, 234110.

[39] M. Ceotto, S. Yang and W. H. Miller, J. Chem. Phys. 2005, 122, 044109.

[40] T. Yamamoto, H. Wang and W. H. Miller, J. Chem. Phys. 2002, 116, 7335.

[41] J. W. Tromp and W. H. Miller, Faraday Discuss. Chem. Soc, 1987, 84, 441.

[42] E. Sim, G. Krilov and B. J. Berne, J. Phys. Chem. A 2001, 105, 2824.

[43] S. Bonella, M. Monteferrante, C. Pierleoni, and G. Ciccotti, J. Chem. Phys. 2010, 133, 164104.

[44] Q. Shi and E. Geva, J. Phys. Chem. A 2004, 108, 6109.

[45] I. R. Craig and D. E: Manolopoulos, J. Chem. Phys. 2005, 122, 084106.

[46] E. Pollak and E. Martin-Fierro, J. Chem. Phys. 2007, 126, 164107; E. Martin-Fierro and E. Pollak, J. Chem. Phys. 2006, 125, 164104.

[47] W. Zhu and Y. Zhao, J. Chem. Phys. 2008, 129, 184111.

[48] S. Bonella, D. Montemayor, and D. F. Coker, PNAS 2005, 102, 6715.

[49] N. Markovic and J. A. Poulsen, J. Chem. Phys. A 2008, 112, 1701.

[50] T. Seideman and W. H. Miller, J. Chem. Phys. 1992, 96, 4412; 1992, 97, 2499. 
[51] U. Manthe and W. H. Miller, J. Chem. Phys. 1993, 99, 3411.

[52] Y. Guo, D. L. Thompson and W. H. Miller, J. Phys. Chem. 1999, 103, 10308.

[53] W. H. Miller, Y. Zhao, M. Ceotto, and S. Yang, J. Chem. Phys. 2003, 119, 1329; M. Ceotto and W.H. Miller, J. Chem. Phys. 2004, 120 (14), 6356.

[54] D. J. Tannor and S. Garashchuk, Annu. Rev. Phys. Chem. 2000, 51, 553.

[55] S. Garashchuk and T. Vazhappily, J. Chem. Phys. 2009, 131, 164108.

[56] R. van Harrevelt, G. Nyman, and U. Manthe, J. Chem. Phys. 2007, 126, 084303 ; T. Wu, H.-J. Werner, and U. Manthe, Science 2004, 306, 2227.

[57] R. Landauer, IBM J. Res. Dev. 1957, 1, 223.

[58] S. Datta, "Quantum Transport: Atom to Transitor", Cambridge University Press, Cambridge (UK), (2005); S. Datta, "Electronic Transport in Mesoscopic Systems (Cambridge Studies in Semiconductor Physics and Microelectronic Engineering)", Cambridge University Press, Cambridge (UK), (1995); R. Kim, S. Datta, M. S. Lundstrom, J. Appl. Phys. 2009, 105, 034506.

[59] Here we prefer to adopt the integration at the place of the sum, since the spectrum is continuos far from the scattering interaction region

[60] E. Pollak and W. H. Miller, Phys. Rev. Lett. 1984, 53, 115.

[61] B. Poirier, Chem. Phys. 2010, 370, 4; J. Schiff and B. Poirier, J. Chem. Phys. 2012, 136, 031102.

[62] Wolfram Research, Inc., Mathematica, Version 8.0, Champaign, IL 2010.

[63] See Supplementary Material Document No. for the Mathematica source file and its detailed description.

[64] D. Colbert and W. H. Miller, J. Chem. Phys. 1992, 96, 1982.

[65] H. Jeffreys, Proc. Lond. Math. Soc. 1923, 23, 428; G. Wentzel, Zeitschrift für Physik 1926, 38, 518; H. A. Kramers, Zeitschrift für Physik 1926, 39, 828; L. Brillouin, Comptes Rendus de l'Academie des Sciences 1926, 183, 24.

[66] The $\lim _{\Gamma \mapsto 0} \int_{0}^{+\infty} e^{-\beta E} N(E) d E=0$, however we are never taking the limit since $\Gamma$ is finite and the Dirac-delta is employed as an approximation to the Breit-Wigner distribution.

[67] W. H. Miller, J. Chem. Phys. 1968, 48, 1651.

[68] W. H. Miller, private communication 